\title{
Harvesting the wind energy through an actively controlled pitch-plunge flapping wing
}

\author{
F. Frunzulica ${ }^{1,2}$, A. Dumitrache ${ }^{2}$, M. Stoia ${ }^{1}$, I. Predoiu ${ }^{1}$ and B. Suatean ${ }^{1}$ \\ ${ }^{1}$ Department of Aerospace Engineering \\ POLITEHNICA University of Bucharest \\ Polizu Street 1-6, RO-011061 Bucharest, Romania \\ Phone/Fax number:+40722894399/+4021 318 2433, e-mail: ffrunzi@yahoo.com \\ 2 "Gh. Mihoc - C. Iacob" Institute of Mathematical Statistics and Applied Mathematics, \\ Calea 13 Septembrie 13, 050711 Bucharest, Romania \\ Phone/Fax number+4021318 2439 /+4021 318 2433, e-mail: alexandru.dumitrache@ima.ro
}

\begin{abstract}
A different approach for harvesting the wind energy with an actively controlled pitch-plunge aeroelastic system is investigated. The paper present some theoretical considerations and results regarding an oscillating wing. The performances of the flapping wing system were investigated numerically and experimentally for the power extraction process.
\end{abstract}

\section{Key words}

Wind energy, Flapping wing, Fluid-structure interaction, Active control

\section{Introduction}

Compared to the conventional systems using wind turbines which are based on transforming the wind energy into mechanical energy of a rotational horizontal or vertical axle rotor, the new proposed systems under consideration convert the wind energy into mechanical energy too, but of a coupled translation-rotation (plunge-pitch) motion. This mechanical energy can be valued using one or more generators. The pitch-plunge motion may be passively or actively controlled by the driving elastic system and by flap and/or slat controls (to maintaining the self-oscillation phenomenon).

Gaining energy from an air flow is a well known (aeroelastic) phenomenon for the aircraft engineers [1]. Taking into account an aerodynamic profile freely oscillating in two directions (one bending or translation and one rotation, referring to a fixed point within the profile), and if between the bending motion $(w)$ and the rotation motion $(\alpha)$ there is a $90^{\circ}$ shifting when lift is oriented along the motion, than the work completed by the profile during the entire cycles is positive, thus the profile is extracting energy from the air flow. The phenomenon is known as flutter and occurs on lifting surfaces under specific conditions reflected by the structural bending and torsion elasticity [1] (Fig. 1).
An oscillating pitch motion creates an oscillating aerodynamic force on the airfoil, which then plunges in response. Existing flapping systems extract power from the oscillatory plunging motion of the airfoil. Two technical possibilities are available:

a) the pitching motion is either prescribed (e.g., [2], in which case some of the power extracted from the plunge motion is used to drive a pitch motor, and the pitch plunge motion becomes periodically) or

b) entirely passive (e.g., [3], in which the coupling pitchplunge motion is determined directly by a fluid-structure interaction problem).

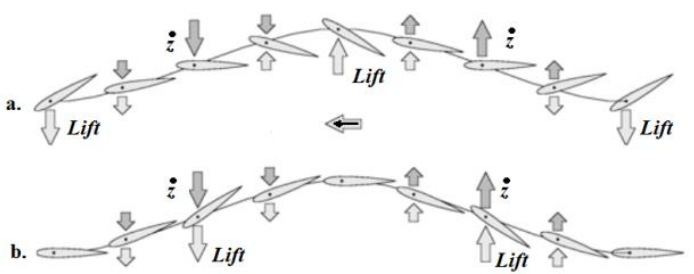

Fig. 1 The flutter phenomenon (net mechanical work $\approx 0$ in the case a., and $>0$ in the case b.)

The efficiency of such new type wind energy harvesting systems is remarkable: the maximum power coefficient reaches the maximum value of 0.5 in the case of the quasi-stationary oscillation mode and for nonlinear oscillation mode (as in the case of multi-plane configurations) the values can be greater than $0.7-0.8$ [4].

Further, the proposed system has the following advantages: the manufacturing of the blade is simple (straight blades); the phenomenon of self-oscillation will increase the amount of energy extracted from the wind; the use of an elastic support system (mechanical) which 
allows for self-oscillating; the axis of the system is vertical, the weight of the oscillating blade being supported by a passive magnetic suspension system (a solution to reduce the friction in the supporting system); the use of passive systems to amplify the self-oscillating phenomenon to obtain large magnitudes required for increased energetic efficiency; a simple mechanical system used for transmitting of the motion to the generator (linear or rotational generator); a system for direct active control (the evaluation of the system being assessed using accelerometers and/or displacement sensors); the state estimator and the controller will be designed in such a way that the functionality of the systems is assured; the control is implemented with an adaptive microprocessor/ microcomputer.

Among the drawbacks of these systems we mention that they have been known to suffer from mechanical fatigue, they can be damaged in storms and are unable to function in bad weather. This may build up the maintenance costs. Furthermore, it is not yet clear the noise level and the impact on the environment (for instance, the public often complains of the appearance and noise of classical wind turbines located near their homes and/or businesses).

In recent decades there are a number of concerns regarding the exploitation of these oscillating systems to extract energy from a stream of air or water [5] or another similar systems [7-10].

In this paper we present a theoretical and background necessary to develop an oscillating system with two degrees of motion, i.e. plunging and pitching, able to harvest the wind energy with a good efficiency.

\section{Oscillating System with two DOF}

The basic system with 2 degrees of freedom is presented in Fig. 3, where notations are those in standard textbooks on Aeroelasticity: $A C$ - aerodynamic center, $E$-elastic center, $K_{w^{-}}$"plunge"-suspension stiffness constant, $K_{\alpha}$-"pitch"suspension stiffness constant, $S_{\alpha}$-model mass static moment about $E$, $M$-model mass, $L$-lift, $M_{E^{-}}$aerodynamic moment in $\mathrm{E}, U$-air velocity, etc..

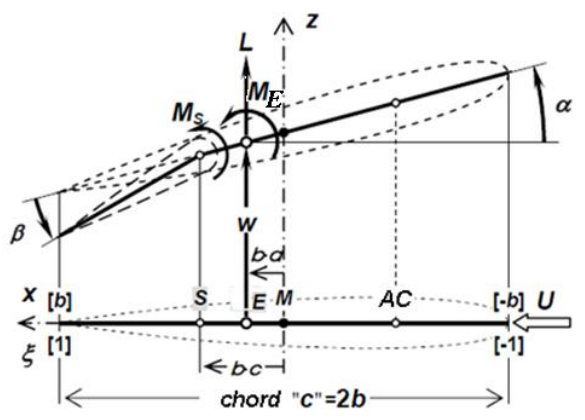

Fig. 2 Oscillating system with 2 degrees of freedom

The governing equations of this two-degree of freedom (DOF) system (including the effect of a flap deflection) can be written [1] in matrix form as - recall that both $w(t)$ and $\alpha(t)$ denote displacements from an initial equilibrium state:
$\left[\begin{array}{cc}M & -S_{\alpha} \\ -S_{\alpha} & I_{\alpha}\end{array}\right] \cdot\left\{\begin{array}{l}\ddot{w} \\ \ddot{\alpha}\end{array}\right\}+\left[\begin{array}{cc}C_{w} & 0 \\ 0 & C_{\alpha}\end{array}\right] \cdot\left\{\begin{array}{l}\dot{w} \\ \dot{\alpha}\end{array}\right\}+\left[\begin{array}{cc}K_{w} & 0 \\ 0 & K_{\alpha}\end{array}\right] \cdot\left\{\begin{array}{l}w \\ \alpha\end{array}\right\}=\left\{\begin{array}{l}L \\ M_{E}\end{array}\right\}$

or in matrix form

$\mathbf{M} \dddot{\mathbf{q}}+\mathbf{C} \dot{\mathbf{q}}+\mathbf{K q}=\mathbf{Q} ; \quad \mathbf{Q}=\mathbf{A}_{0} \mathbf{q}+\mathbf{A}_{1} \dot{\mathbf{q}}+\mathbf{A}_{2} \ddot{\mathbf{q}}+\mathbf{B}$

The motion of the system can be:

a) unsteady periodic; for this case, the displacements are periodic time-dependent $w(t)=w_{0} e^{j \omega t}, \alpha(t)=\alpha_{0} e^{j \omega t}$ and the problem depends to the calculus of the periodic aerodynamic forces. The incompressible regime is a remarkable case for that exist relative simple solutions (e.g. Theodorsen method [11] - Fig. 2). The Theodorsen's method assumes that the aerodynamic forces can be written as a sum of circulatory and noncirculatory components (incompressible inviscid flow):

$$
\begin{aligned}
L & =L_{n c}+L_{c}=\pi \rho b^{2}(-\ddot{w}+U \dot{\alpha}-b a \dot{\alpha})+ \\
& +2 \pi \rho U b[-\dot{w}+U \alpha+b(1 / 2-a) \dot{\alpha}] \cdot C(k) \\
M & =M_{n c}+M_{c}= \\
& =\pi \rho b^{2}\left[-b a \ddot{w}-U b(1 / 2-a) \dot{\alpha}-b^{2}\left(1 / 8+a^{2}\right) \ddot{\alpha}\right]+ \\
& +2 \pi \rho U b^{2}(1 / 2+a)[-\dot{w}+U \alpha+b(1 / 2-a) \dot{\alpha}] \cdot C(k)
\end{aligned}
$$

where $k=\omega b / U$ is the reduced frequency and $C(k)$ represents the Theodorsen complex function. The effective angle of attack that contributes to the circulatory part of the aerodynamic forces is given by:

$$
\alpha_{e f f}=\left[\alpha-\frac{\dot{w}}{U}+\frac{b}{U}\left(\frac{1}{2}-a\right) \dot{\alpha}\right] \cdot C(k)
$$

b) quasi-steady; this motion corresponds to small reduced frequency, $k \cong 0.05$. The aerodynamic model neglects influence of downwind vortices, and thus $C(k) \rightarrow 1$ when $k \rightarrow 0$.

c) unsteady arbitrary.

The flutter condition using the root locus for a generic motion can be shown for a generic motion described by the relations: $w(t)=w_{0} \sin (\omega t), \alpha(t)=\alpha_{0} \sin (\omega t+\pi / 2)$

Obviously, exceeding the critical conditions of flutter, leads to the damaging or even destruction of the structure. In order to avoid such an occurrence, the active control methods have to be used to eliminate the flutter. This is done using certain automated devices. These devices control the natural instability of the structure which delays the occurrence of the flutter at a critical.

The main challenge is the design a self-oscillating wing system, actively controlled to performed a convenient pitch-plunge motion at different flow speeds and able to extract and convert the wind energy into electrical energy by means of a linear and/or angular electrical generator(s).

\section{Motion Control}

The control is required to ensure boundary cycle type oscillation at speeds higher than $U_{F}$ (natural flutter speed 
of the aeroelastic system); ideally it is required to have a very low flutter speed $U_{F}$ to enable the benefit of a larger wind speed spectrum. It is understood that the selection of a proper $U_{\text {des }}$ (design speed) enables the system operation also at high wind speed (over $35 \mathrm{~m} / \mathrm{s}$ ), providing an advantage over the classic wind power systems.

The oscillating system will be controlled by a programmable controller or by a portable computer, the control law being implemented interconnecting and tuning a direct integration connections block scheme (a usual scheme in aero-servo-elasticity), where the motion sensors could be as well motion transducers or accelerometers.

We propose a control system similar but not identical to that used in classic flutter suppression. This system is based on the flap motion control (the flap works as an antiaeroelastic vibrations actuator) and it has been tested in our Fluid Mechanics Laboratory (Fig. 3) from Faculty of Aerospace Engineering - POLITEHNICA University of Bucharest $[12,13]$.

Then the control law is adapted in order to maintain a desired oscillation at airflow velocities greater than $U_{F}$.
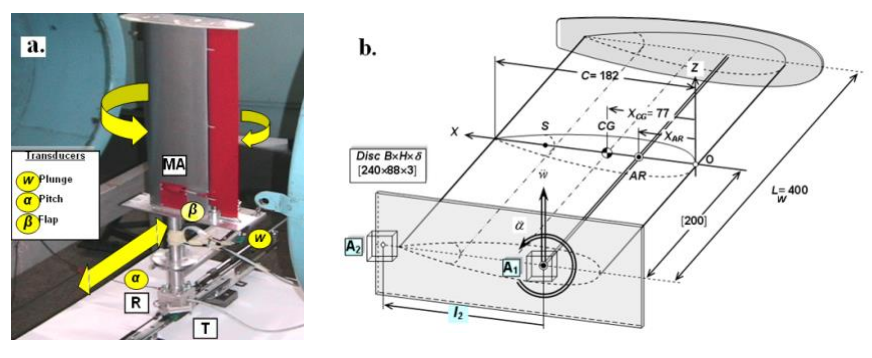

Fig. 3 Aero-servo-elastic model: (a) Experimental model installed in the wind tunnel (T-translation, R-rotation, MA-flap driving) and (b) The geometry of the wing model.

The system of two second-order differential equations (1) can be represented into a state-space form. We choose the "natural" states

$$
\mathbf{x}=\left[\begin{array}{llll}
w(t) & \alpha(t) & \dot{w}(t) & \dot{\alpha}(t)
\end{array}\right]^{T}
$$

We consider that the motion are unsteady periodic, and from the system (2) we retain, in the right, member the derivatives to order one:

$$
\mathbf{Q}=\mathbf{A}_{0} \mathbf{q}+\mathbf{A}_{1} \dot{\mathbf{q}}+\underbrace{L_{W} \rho U^{2} b\left[\begin{array}{l}
C_{L}^{\beta} \\
2 b C_{M, E}^{\beta}
\end{array}\right]}_{\mathbf{B}^{\prime}} \cdot \beta(t)
$$

Thus, the canonical system can be written in the following form:

$$
\dot{\mathbf{x}}=\mathbf{A}_{\mathrm{wa}} \mathbf{x}+\mathbf{B}_{\beta} \beta(t)
$$

The conventional flutter problem is formulated by simply discarding the inhomogeneous part in (7), that is through building the system "free dynamics"

$$
\dot{\mathbf{x}}=\mathbf{A}_{\mathbf{w a}}(U, \rho) \cdot \mathbf{x}
$$

For practical applications (on flutter suppression and to control oscillations of the system), one must take into account the dynamics of the command line (actuator) [14].

\section{A. The "direct connection" scheme}

Implicit in the full-state feedback concept is the condition of full access to all states. Since in the proposed set-up accelerometers are used as sensors, the state vector (displacements and velocities) must be built from measured accelerations. In this respect, an attempt has been made to "construct" the states by simply successively integrating the accelerations (Fig. 4).

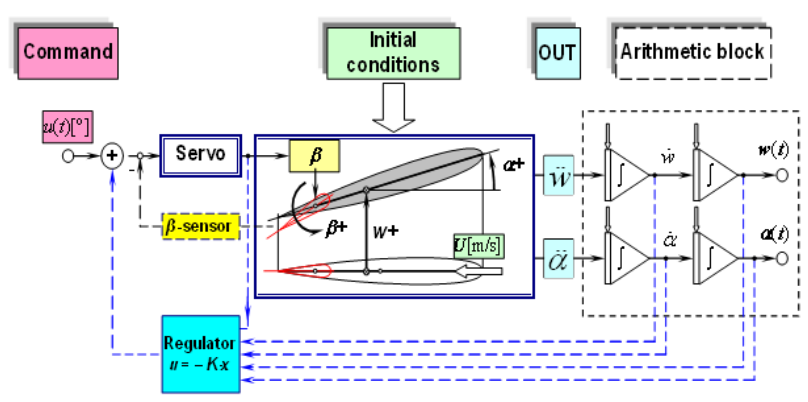

Fig. 4 System with controller - The "direct connection" scheme.

\section{B.The state estimator}

The alternative to the former is the observer, a "pure mathematical" device through which the states are being reconstructed from the output (Fig. 5).

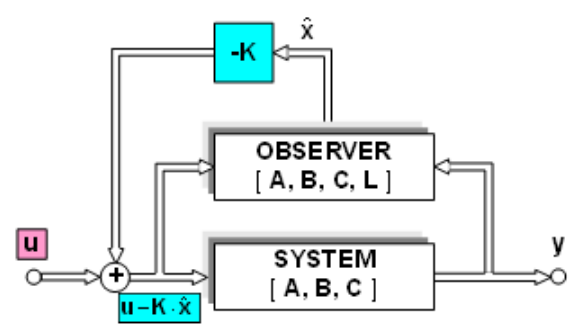

Fig. 5. System plus observer

For the control of the above mentioned demonstrator model, a simple electrically driven trailing-edge flap was added over the entire wing span. For tracking the plunge/pitch system motion, a couple of linear/angular transducers have been mounted on wing. Further, a flap deflection angular sensor has been provided in order to have a precise control on this latter's movement.

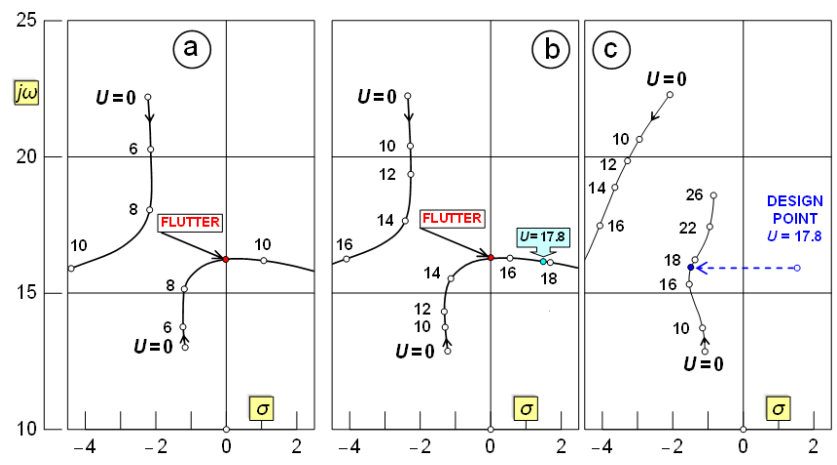

Fig. 6 System with controller - The "direct connection" scheme.

The parameters for the experimental aero-servo-elastic model are: wing chord $(C=2 b=0.182 \mathrm{~m})$, wing span $\left(L_{W}=0.4 \mathrm{~m}\right)$, rotation axis $-C E(\sim 28 \% C)$, mass centre of 
the model $-C G(\sim 42 \% C)$, flap hinge line $-S(\sim 70 \% C)$, model mass $(M=1.855 \mathrm{~kg})$, model mass static moment about $C E\left(S_{\alpha}=0.01729 \mathrm{~kg} \cdot \mathrm{m}\right)$, model mass moment of inertia about $C E \quad\left(I_{\alpha}=2.745 \cdot 10^{-3} \mathrm{~kg} \cdot \mathrm{m}^{2}\right)$, "plunge"suspension stiffness constant $\left(K_{w}=321.6 \mathrm{~N} / \mathrm{m}\right)$, "pitch"suspension stiffness constant $\left(K_{\alpha}=1.25 \mathrm{Nm} / \mathrm{rad}\right)$, damping coefficients as a fraction of critical damping $\left(\zeta_{w}=0.1\right.$, $\left.\zeta_{\alpha}=0.1\right)$.

The tests performed in the wind tunnel have shown the model to enter a violent flutter at exactly $17.8 \mathrm{~m} / \mathrm{s}$ and $\sim 17$ $\mathrm{rad} / \mathrm{s}$ frequency [13]. These correspond to a reduced frequency of about 0.09 . Now, the system "free dynamics" (15) has been simulated through the Laplace transform and the root-locus (RL) procedure (Fig. 6) with the basic mechanical parameters determined experimentally.

\section{Numerical Simulation}

As we have seen in previous sections, the classically models (e.g. Theodorsen's model) does not include viscous and other nonlinear aerodynamic effects. A more relevant information (e.g. effect of the drag and of the dynamic stall) can be obtained through numerical simulations, i.e. using a CFD code [15].

To investigate the energy harvesting from a flow, we have considered a NACA0012 airfoil with a chord length $c=15$ $\mathrm{cm}$ which performs a coupled plunge-pitch oscillation (the pitching motion around the point located at $1 / 4 c$ from the leading edge) described by

$$
h(t)=H \cdot \sin \left(\omega_{h} t\right), \quad \alpha(t)=-\Theta \cdot \sin \left(\omega_{\alpha} t+\Delta \phi\right) \cdot \frac{\pi}{180}
$$

where

$$
\begin{aligned}
& H=0.1 \mathrm{~m}, \Theta=25^{0}, \omega_{h}=\omega_{\alpha}=18.67 \mathrm{rad} / \mathrm{s}, \\
& \Delta \phi=\pi / 2, k=\omega c /(2 U)=0.1
\end{aligned}
$$

The airfoil is placed in a free uniform flow with velocity $V_{\infty}=14 \mathrm{~m} / \mathrm{s}$ and turbulence intensity of about $5 \%$, which corresponds to a Reynolds number $\operatorname{Re}=1.35 \times 10^{5}$. The computational domain is composed by an inner oscillating circular domain and a exterior circular domain with boundary at $25 \mathrm{c}$. The inner domain executes a rigid pitching motion around its centre with the angular velocity $\dot{\alpha}(t)$, and plunge (in the exterior domain) with velocity $\dot{h}(t)$ (Fig. 7).

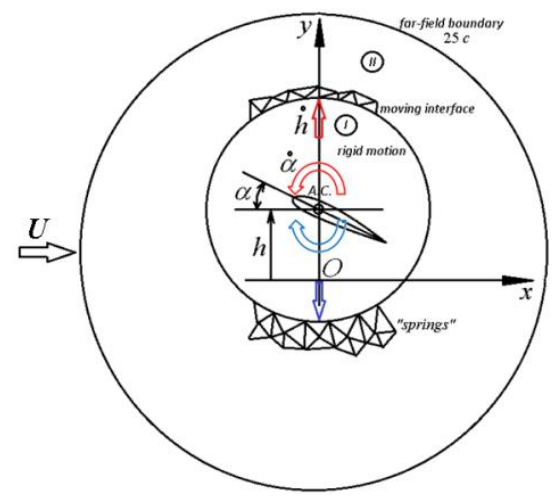

Fig. 7 System with controller - The "direct connection" scheme.
For the present study, the Unsteady Reynolds Averaged Navier-Stokes (URANS) model is the suitable approach to perform the simulation with an acceptable computational cost and, at least, reasonable accuracy. Ansys Fluent code for computational simulations has been used [15]. The $k-\omega S S T$ turbulence model was used in simulation $\left(\mathrm{Re}=1.45 \times 10^{5}\right)$. The numerical time step is set to be $0.0004 \mathrm{~ms}$ based on the characteristic time flow; after four complete oscillations the solution became periodical.

In Fig. 8 is shown the snapshots of the streamlines and pressure contours $\left(p-p_{a t m}\right)$ for $t=0, T / 8, T / 4$, and $T / 2(s)$ (the last cycle of oscillation). The coupling between pitch and plunge motion decrease maximum effective incidence and dynamic stall effect can be reduced. This is an advantage because we can increase the maximum pitch angle to increase lift in oscillating process.

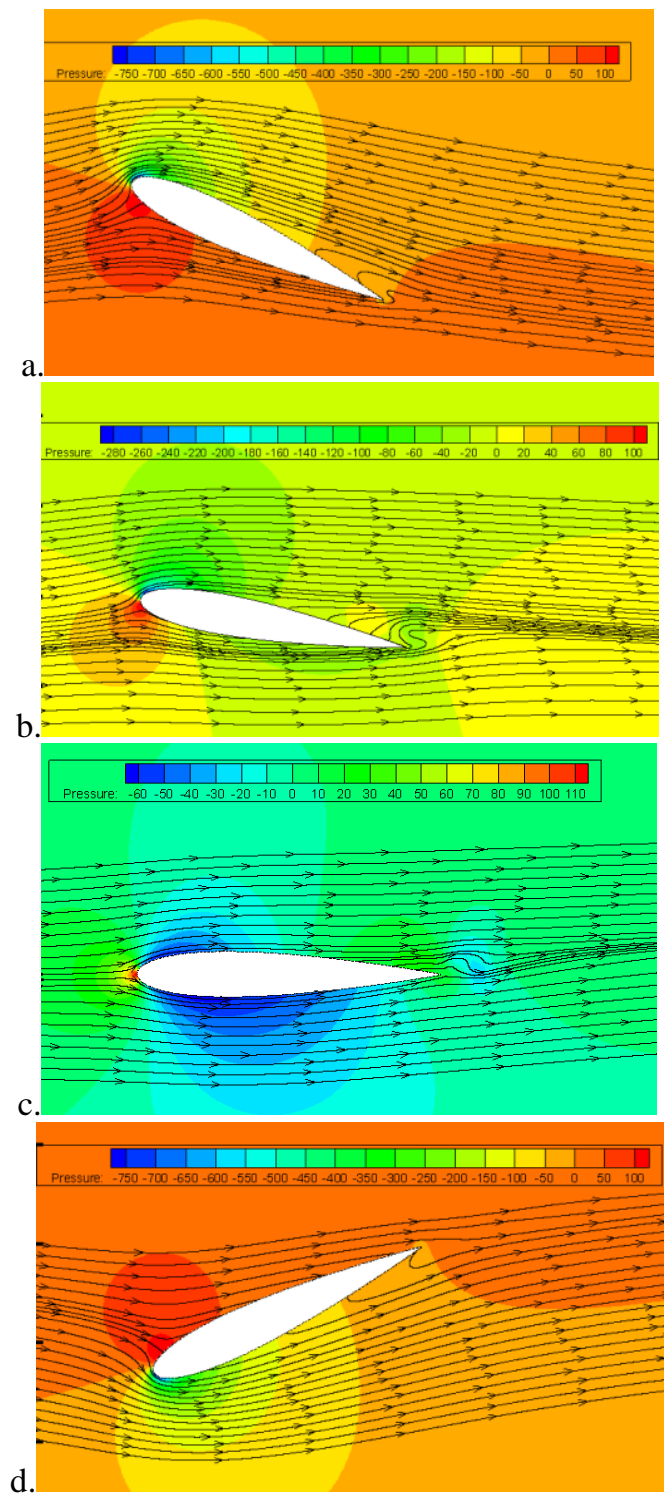

Fig. 8 Streamlines and pressure contour at $0, T / 8, T / 4$, and $T / 2(s)$; period of oscillation $\mathrm{T}=0.337 \mathrm{~s}$.

In Fig. 9 is shown the variation of the lift and aerodynamic moment; we observe that the values of the aerodynamic moment is about with two order less than 
lift, and thus the contribution of the pitching motion to the extracting power from airflow is negligible.

Fig. 12 depicts the contribution of pitching and plunging motion to the power extracted from the airflow.

The power extracted over a period is calculated with relation:

$$
\begin{aligned}
& \bar{P}_{t}=\frac{1}{T} \int_{0}^{T}[L(t) \cdot \dot{h}(t)+M(t) \cdot \dot{\alpha}(t)] d t=22.884 \mathrm{~W}, \\
& C_{P}=\frac{\bar{P}_{t}}{1 / 2 \rho U^{3} S}=0.091
\end{aligned}
$$

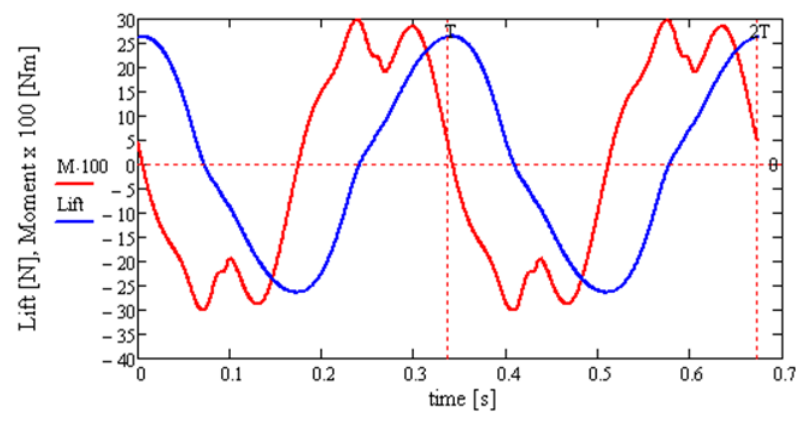

Fig. 9 Aerodynamic forces on oscillating airfoil.

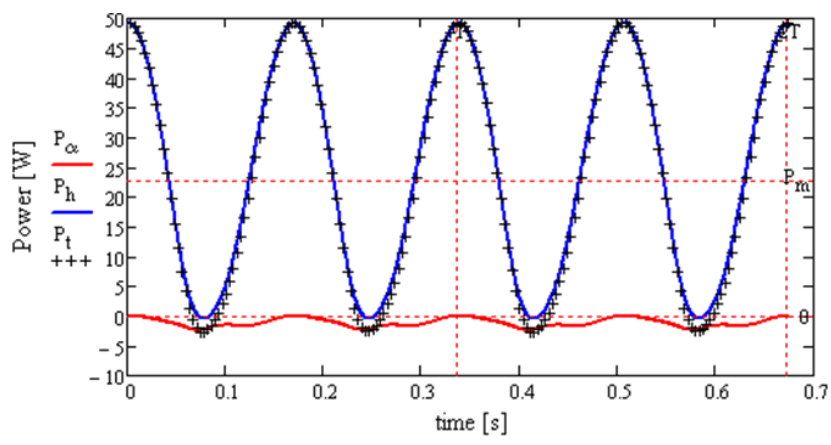

Fig. 10 Power of the oscillating system.

\section{Conclusion}

In this work we have investigated the possibility to harvest the wind energy with an actively controlled pitch-plunge aeroelastic system. First, the active control is used here not to damp the aeroelastic vibrations but to maintain an oscillatory motion of the aeroelastic device for a desired interval of wind velocities. After then, in order to improve the prediction capabilities during the design process, mainly for the efficiency of the system, we have developed a computational methodology based on a CFD approach for the power extraction process. The work is under progress and the next step is to couple the CFD tool to a structural solver, for an accurate prediction of the aeroelastic vibrations of the system.

\section{Acknowledgement}

This work was realized through the Partnership programme in priority domains - PN II, developed with support from ANCS CNDI - UEFISCDI, project no. PNII-PT-PCCA-2011-3.2-167.

\section{References}

[1] A. Petre, Aeroelasticity Theory - Periodic dynamic phenomena, Romanian Academy Publishing House, Bucharest, 1973.

[2] Q. Zhu, M. Haase and C. H. Wu, "Modeling the capacity of a novel flow-energy harvester," Applied Mathematical Modeling, vol. 33, no. 5, pp. 2207-2217, 2009.

[3] Z. Peng and Q. Zhu, "Energy harvesting through flowinduced oscillations of a foil," Physics of Fluids, vol. 21, no. 12, Paper 123602, 2009.

[4] http://www.windturbine-performance.com/

[5] W. McKinney and J. DeLaurier, "The wingmill: An oscillating wing windmill," Journal of Energy, vol. 5, no. 2, pp.109-115, 1981

[6] http://www.econologica.org/index.html

[7] http://w2energycorp.com/home

[8] K.D. Jones, S.T. Davids and M.F. Platzer, "Oscillatingwing power generation," ASME Paper FEDSM 99- 7050, [3rd ASME/JSME Joint Fluids Engineering Conference, July 1999].

[9] K.D Jones, K. Lindsey and M.F. Platzer, "An Investigation of the fluid-structure interaction in an oscillating-wing micro-hydropower generator," in Fluid-Structure Interaction II, Wessex Institute of Technology Press, 2003, pp.73-82.

[10] M.F. Platzer, M.A. Ashraf, J. Young and J.C.S. Lai, "Extracting power in jet streams: pushing the performance of flapping wing technology," 27th International Congress of the Aeronautical Sciences, Nice, France, 2010, pp. 1924

[11] R.L Bisplinghoff,.H. Ashley and R.L. Halfman, Aeroelasticity, Addison-Wesley, Cambridge, Mass, 1955.

[12] I. Predoiu, F. Frunzulica and M. Stoia-Djeska, "Refined model of a flutter suppression system with practical assessment," 10th Conference on Dynamical Systems Theory and Applications, December 7-10, 2009. Łódź, Poland (Conference Proceedings).

[13] Theoretical and experimental studies regarding the damping of aeroelastic oscillation through active control techniques, AEROSPATIAL - National Research Program (2004-2006).

[14] B. S. Liebst, "Accelerometer placement in active flutter suppression systems," Journal of Guidance and Control, vol. 10 , no. 5 , Sept.-Oct. 1987 , pp. 441-446.

[15] *** Ansys Fluent 13. User's guide. Fluent documentation. 